\title{
Chronic Kidney Disease Diagnosis Model Based on Case Based Reasoning
}

\author{
Ermiyas Birihanu Belachew, Hailemichael Kefie Tamiru
}

\begin{abstract}
Provision of health care services is still a major challenge for developing countries. To mention some of the challenges: Lack of highly qualified medical human resources, financial as well as the ability of manage and transform scare resources to meet healthcare needs. In particular, In Ethiopia health care management related to the kidney disorder suffers from the following challenges: lack of highly qualified medical human resources, financial as well as the ability to manage and transform scarce resources to meet healthcare needs. On the one hand, Artificial Intelligence (AI) helps the medical sciences. Hence, in this paper we proposed a framework for CBR system to facilitate and support the diagnosis of chronic kidney diseases with domain expert's advice. Interview and techniques have been employed on this study to acquire the necessary information required to develop intended CBR system. Finally, we evaluate the performance of the developed framework using recall and precision.
\end{abstract}

Keyword: Case basereasoning, Preprocessing, framework, Kidney disorder

\section{INTRODUCTION}

In the past time, an examination of sicknesses has been for the most part dependent on creature models, which are believed to be unavoidable without better options. As a result of progression of clinical database that contains data about clinical information gathered from increased care patients have made the Data-Driven methodology an attractive alternative for the examination of humanillnesses. Numerous old models can be confirmed and new inquiries can be addressed utilizing the information. The significant confinement is that the information is gathered among the conveyance of consideration, without explicit research purposes. In this manner, not all demonstrating errands are plausible with this information. The early detection of renal impairment is therefore important to allow for the institution of appropriate diagnostic and therapeutic measures, and potentially maximize protection of residual renal function in the setting of kidney disease. Monitoring the progression disease to therapy.

According to World Health Organization (WHO) report healthcare services is a major challenge for most developing countries. Some of these challenges are lack of highly qualified medical human resources, financial as well as the ability to manage and transform scarce resources to meet healthcare needs[1] .

Revised Manuscript Received on December 30, 2019.

* Correspondence Author

Ermiyas Birihanu Belachew*, Lecturer, Software Engineering, Wolkite University, Wolkite, Ethiopia

Hailemichael Kefie Tamiru, Lecturer, Software Engineering, Wolkite University, Wolkite, Ethiopia

(C) The Authors. Published by Blue Eyes Intelligence Engineering and Sciences Publication (BEIESP). This is an open access article under the CC BY-NC-ND license (http://creativecommons.org/licenses/by-nc-nd/4.0/)
Chronic diseases, such as heart disease, stroke, cancer, chronic respiratory and more which are the leading factor for mortality in the world; $63 \%$ mortality [2]and in Ethiopia. According to 2016 WHO data, renal disease deaths in Ethiopia accounted for $1.47 \%$ or 12,038 of total deaths [3]. Thus, this invisible epidemic of chronic diseases slows down the economic development of many countries and contributes to poverty for those country, one of the challenge face by this issue are Ethiopia.

According to WHO report in 2006, financial resources for kidney diagnosis healthcare in Ethiopia are extremely insufficient [4] ; a renal (kidney) unit was opened in the TikurAnbessa Hospital, Addis Ababa, in 1980 with the assistance of a Cuban team from the Institute of Nephrology in Havana. The unit provides outpatient and inpatient services including dialysis. There are presently 6 private dialysis units in Addis Ababa (+ 1 in Adama) with a total of $\sim 50$ HD machines catering for a total of $\sim 180$ patients with end-stage renal failure.

Shortage of nephrologists, their unfair distribution and lack of knowledge among primary healthcare workers are the main challenges that become an obstacle to address kidney disorder health services satisfactorily throughout the country. According to Yewondwossen report, "there are only 9 nephrologists in Ethiopia for a total of 90 million people, However, there are several Ethiopian nephrologists currently practicing in the US and Europe" [5] .

As a result, computer-aided solutions play a prominent role to address the aforementioned challenges. To this end, rulebased and CBR are two of the widely used techniques employed in such settings. As stated in [6], however, rulebased systems cannot learn automatically as new rules are usually inserted manually. On the other hand, CBR minimizes the task of knowledge acquisition and can solve problems using specific knowledge. To the best of the researcher's knowledge, no studies are conducted CBR for kidney disorder diagnosis in Ethiopian context. Thus, in this study an attempt to apply CBR for kidney disorder diagnosis. The rest of the paper is organized as follow. In section II, we briefly describes related work on Case based reasoning. In section III, we provide in depth description of the main methods and procedure they realizes.In section IV, we report the experimentation result and finally we conclude and forward furthered research direction.

\section{RELATED WORKS}

Much et al [7].They proposed master frameworks for the finding of Chronic Kidney Disease. The reason for the exploration is to build up a specialist framework analysis of Chronic Kidney Disease dependent on Mamdani Fuzzy Inference System and decide the dimension of framework precision in finding of Chronic Kidney Disease. 
Akande et al[8], Proposed an electronic master framework for analysis and the executives of kidney infection.

The point of this work is to plan and actualize an online master framework for analysis and the executives of kidney illnesses. For the improvement of master framework, free e2gLite master framework building apparatus (shell) executed as a Java applet was connected which is outfitted with a deduction instrument and an information base, and the web interface was created with the utilization of HTML. The framework poses inquiries of the client to inspire the data required so as to prescribe or give last outcome dependent on the client information and utilizations IFTHEN principles to speak to learning.

The framework proposed by Salem et al[9, 10].ready to give a proper determination for the introduced side effects, signs and examinations done to a heart understanding with the relating conviction factor. It tends to be utilized to fill in as specialist demonstrative right hand and bolster the training for the undergrad and postgraduate youthful doctors.

The framework proposed by Abdel et al[10][9], Provides suggestion for controlling agony and giving side effect alleviation in cutting edge malignancy. It very well may be utilized as a device to help and ideally improve the nature of consideration given for those enduring immovable torment. The framework is exceptionally helpful in the administration of the issue, and its errand is to help the youthful doctors to

\section{MATERIALS AND METHODS}

In the following section we discussed about data collection techniques, prepared data, Experimental tool and its investigation results well.

\section{a) Knowledge based system}

Human being brain can able to store various knowledge in this world with different perspectives, however the research works showed human brain is not fully used. The building block of human brain is called neurons and it is approximately 1011 cells. In order to solve the given problem with in these worlds through declarative and procedural ways, but still now it is a mystery that how does the human brain is working. The Knowledge-Based Systems (KBS), which are a step towards an intelligent system, can be justified when a few individuals have the majority of the knowledge[11].

KBS is a branch of artificial intelligence, which is a computer program that attempts to replicate the reasoning processes of a human expert and it can make decisions and recommendations and perform tasks based on user input[12] . Actually, KBS is one of the computer systems that uses and extract knowledge from data, information and knowledge. The implementation of KBS used heuristic human reasoning to determine techniques, steps and mechanisms, in order to solve problems that do not have a traditional algorithmic solution[13].

\section{b)Knowledge acquisition in identification of cases}

In this study, the researchers passed some basic activities like gathering the required knowledge, analyzing the knowledge, identifying important concepts and attributes (symptoms) and finally modeling it.

During knowledge acquisition determines how knowledge is obtained and where it is obtained determines the usefulness check their determination.

of the system[14] . Knowledge acquisition is referred to as a process of eliciting, structuring and representing knowledge from some knowledge source, usually human experts, in order to build knowledge based system[15] .As indicated by Jones [16]knowledge elicitation and structuring are the two most important activities of knowledge acquisition processes that are carried out by knowledge engineer in order to build knowledge based system.

\section{c) Knowledge Acquisition from Domain Expert}

In this study the primary source of knowledge gathers from domain experts. Domain expert is the a person who is specialist or knowledge like Medical Doctors, Nurses, Health Officers and others in solving problems of domain area, the knowledge of experts play a fundamental role in structuring the knowledge base and developing the knowledge base system. To collect the relevant knowledge the researcher applied semi-structured interview technique. Hence, the basic concerns of this chapter is eliciting required knowledge from the domain experts, two (2) domain experts from each hospital are selected using purposive sampling technique.

As results of this, expert in the profession (Medical Doctor, Health Officer, Nurse and other supportive) were interviewed to obtain the required knowledge of kidney. The interview discussion gives an emphasis the step which is followed by experts in diagnosis the kidney up to their decision making tools (Laboratory Results).

\section{- Knowledge Acquisition from Relevant Document}

The second way of knowledge elicitation the researcher applied in this study was documented analysis; which is a vital one for knowledge gathering in our study. The researcher reviews various significant papers which are related to kidney disorder diagnosis. These documents are: Books like harrsion principles of Internal Medicine 18e and others, Articles that are published in different journals and Ethiopia Standard Treatment Guidelines for General Hospital.

\section{- Knowledge Acquisition from patients}

In addition to the above knowledge acquisition in this study, we also collected from patients in selected hospitals like Jimma, Atate, St Paulos and Hawassa. To gather the required knowledge written questioners was used. Since one of the main focuses of written question with patients is to elicit required knowledge, the researchers were selected the number of patients, according to the magnitude of peoples in the selected hospitals using random sampling techniques. Based on the written questions which was filled by patients responded which are not enough and experienced experts in different hospitals was selected that are able give advice more suitable to each patient.

\section{- Data Set description}

In this study, there were about 90 cases with 28 features that help in the diagnosis of kidney disease. Steps of preprocessing will be applied to these raw data sources to produce high-quality case base enhanced for CKD diagnosis.

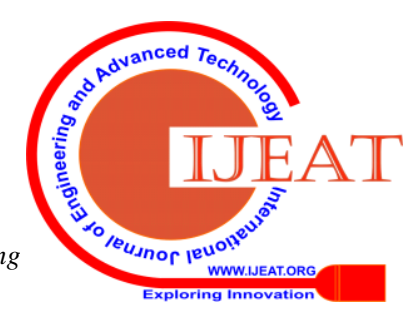




\section{- The proposed case based model}

In this section the researchers focus on design and implementation for the development of workable case based system for chronic kidney disorder.

The basic and relevant case collected from domain experts and patients. Having all the relevant cases and knowledge from the domain expert, patients and different relevant documents, the next task is coding the knowledge into computer using appropriate and efficient knowledge representation methods and tools. For this study, JCOLIBRI 1.1 was used to design and implement the prototype and construct the case structure of recommender system.

To conduct the study more the researchers used nearest neighbor retrieval algorithm to design the retrieval task. This is because of retrieves cases which match partially with the new case and it has the advantage of simplicity in retrieving relevant cases[17] and JCOLIBRI used this algorithm as integrated component for retrieval task.

Figure 1 showed below the working principle for kidney disorder management and the relevant cases were collected from both patients and domain experts from selected hospitals. First, as the new query entered to the prototype of the system matches the new case to the solved case in the case base of the system by using similarity measurement. When there is the relevant cases are found within the case base, then the prototype rank the relevant retrieved cases based on their global similarity. Next the prototype proposed the solution to the query. The proposed solution can be derived directly from a retrieved case that matches exactly or partially to the problem of the new case. But, using the proposed solutions directly may have a risk. Therefore, the user of the system should have made an adaptation by altering the differences between the proposed case and the new case. In addition to adaptation, case inconsistencies are revised if the retrieved case is not the same as the new case. Finally, the revised solution is retained in the case base for future problem solving.

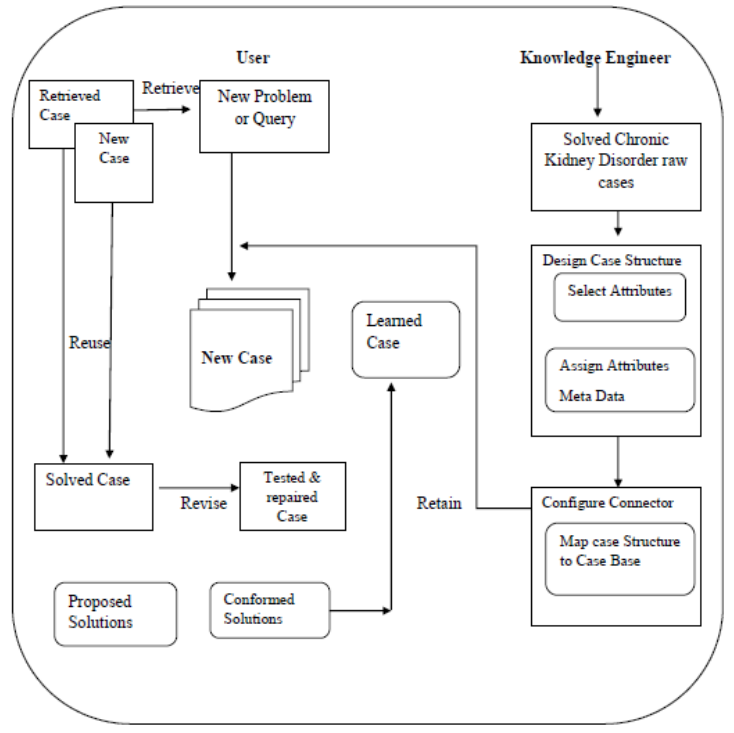

Figure 1: Architecture of Case Based Reasoning for Chronic Kidney Disorder Management

\section{EXPERIMENTATION AND RESULT}

So as to build up the Reasoning system the researchers passed various advances like gathering cases, demonstrating an appropriate case representation, characterizing an exact closeness measure, executing recovery usefulness, and actualizing User Interface (UIs). JCOLIBRI has been built as center modules to propose the essential usefulness for creating case base thinking just as case based analysis framework. Building up another case base conclusion framework was made by composing couple of Java classes that broaden classes of the system and arrange some XML records. To begin the JCOLIBRI graphical User Interface (GUI) application device, dispatch the principle window by tapping on JColibriGUI.bat document and it ends up prepared to use as appeared.

Building the Case Base

As exhibited destinations of this examination, one of explicit goals was building case base involving gathering constant kidney patients cases to give the most significant comparable cases to help area specialists and scientists to distinguish the kidney cases during their clinical occasions. The gathered cases are used to build the case based framework for incessant kidney issue that is fundamental to help space specialists and scientists to distinguish early those cases which is connected with kidney. The case base is existing as a plain content comprises of $\mathrm{N}$ sections speaking to case traits (A1, A2, A3, .., AN) and every $\mathrm{M}$ lines speaking to individual cases $\mathrm{C}(\{\mathrm{C} 1, \mathrm{C} 2, \mathrm{C} 3, \ldots, \mathrm{CM}\})$ each property has an arrangement of potential qualities related to every segment characteristic $A=\{V 1, V 2, V 3, \ldots, V k\}$.

\section{- Case Representations}

The case representation is built such that effectively fit to jCOLIBRI. During planning of such case structure depicts the properties accessible in the cases and used to quantify the similitude between close by cases and the new case (inquiry). The general use of this experimentation is to recover comparative cases to the question from the case base that can direct space specialists, taking care of issues of perplexity and changing an answer for the present issues. Case base were organized to make the recovery procedure proficient.

\section{- Overseeing Case Structure}

The most well-known component of JCOLIBRI is overseeing and characterizing case structure so as to effectively get to the framework window. After made a profound discourse with domain experts select credits were added to the depiction windows of case structure and their properties are allocated for each ascribe to the correct side of the window. At that point the program creates a java source code naturally and spared as XML position.

\section{- Managing Connectors}

Once case structures are configured in JCOLIBRI, diagnosis systems must access the stored cases from case base. JCOLIBRI supports both SQL database and plain text file to store its cases base. 


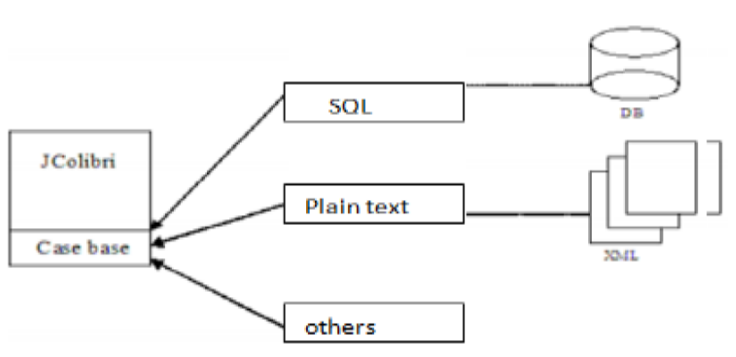

Figure 5: JCOLIBRI Case base schemas

In this study, plain text connector was used as a case base storage. The connector maps the case structure to its column from plain text file which is saved in .txt file format and later saved as XML file like that of case structure

\section{- Managing Tasks / CBR application}

After configuring the connector and case structure, the next task is selecting tasks and methods of application. JCOLIBRI has two types of task packages, namely, Core packages and User defined package tasks. For this study the researcher used core package tasks. A core package contains all classes that represent core functionality of a CBR application such as the domain model, case bases, similarity functions and retrieval algorithms. Core packages also have predefined tasks and methods that used to configure new system by reusing the tasks rather than using tasks or methods defined by the system developer itself like user defined packages, because defined tasks and methods by user itself for every system is time taking and complex. Different core packages are available in JCOLIBRI. The main components of Core packages which are used in CBRISAIAS prototype development are Pre-Cycle, main CBR cycle and Post-Cycle. The component of core packages is the final and important step for creating a new application where the CBR application is configured.

\section{a. Managing Methods}

The overseeing technique library stores classes that really resolve the assignment. These classes can resolve the CBR cycle utilizing in programming or utilizing GUI. All errands that are referenced in 4.3.5.1 ought to have their very own strategies to be allocated so as to accomplish the undertakings objective. Coming up next is a rundown of techniques which are utilized to tackle assignments for this kidney issue application.

\section{b. Evaluation of the Retrieval and Reuse Process by Using Statistical Analysis}

In this examination, recovery of cases is performed utilizing the closest neighbor recovery calculation on the grounds that the execution instrument jCOLIBRI utilizes this calculation. Among recovery, comparative cases are recovered to the new case with proper positioning.

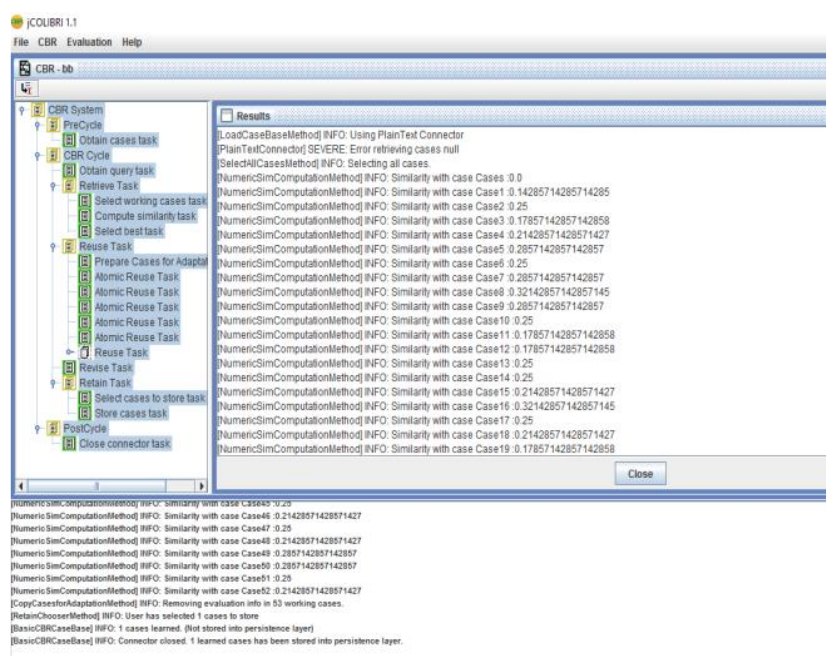

Figure 10: Deploy Case based

Table 1: Depicts Performance Measurement of kidney disorder system using Precision and Recall

\begin{tabular}{|l|l|l|}
\hline Test Case & Precision & Recall \\
\hline Case 55 & 0.41 & 0.57 \\
\hline Case 56 & 0.55 & 0.63 \\
\hline Case 57 & 0.49 & 0.52 \\
\hline Case 58 & 0.34 & 0.45 \\
\hline Case 59 & 0.24 & 0.42 \\
\hline Case 60 & 0.11 & 0.35 \\
\hline
\end{tabular}

As appeared Table 2 both review and accuracy results are better than expected which is a cheerful outcome. The normal review and exactness results $34 \%$ and $55 \%$ separately which is likewise a promising outcome. As found in the Table 2, for each experiment more than normal is enrolled both review and exactness. In any case, in restorative area accomplishing more than normal review and exactness isn't sufficient. Regarding review this examination accomplished a generally excellent outcome.

\section{- Case Revision and Solution Adaptation Testing}

In medical diagnosis adaptation is a commonly required task. Since this study primary objective is creating kidney issue finding framework, adjustment is essential. The reason testing adjustment of arrangements is to assess the framework capacity to reuse cases from the case base. Initially the system load case bases at the PreCycle stage and then selects working cases from the case base and stores the cases in to current context at the retrieval stage.

In general, the adaptation process of kidney issue application is effective as the case highlights of the past and new case have comparative or less irregularity trait esteems. Then again, no adjustment procedure can be executed as the quality estimations of the past and new cases have increasingly disparate or very surprising from the past cases. In any case, regularly an immediate utilization of an unsure arrangement is inconceivable because of the relating dangers, particularly in medicinal finding frameworks. 


\section{- Testing Case Retaining}

Case retaining is the last cycle in kidney thinking application which is a significant advance in putting away new cases which will use for future determination. Particularly, in kidney issue conclusion, holding cases after some time is significant in light of the fact that for the most part the medications are psychotherapy which is generally utilizing specialists and analysts inferred learning and individual experience.

Therapeutic CBR frameworks ought to be structured as long lasting learning application. CBR frameworks in medication ought not be structured uniquely to reuse past scenes with little adjustment rather holding new cases for taking care of comparable issues for what's to come is essential since drug is a quickly changing field occasionally, and medicinal practice rules are consistently refreshed. Prescriptions change, and new medications rise always.

\section{CONCLUSION AND RECOMMENDATION}

In this study, the researcher presented a case based reasoning system for decision support in the health domain particularly on kidney disorder. The system uses past cases (situations) to solve a new case (situation), which was created on the basis of user input.

The main goal of this research was to develop a prototype case based system that can assist the medical students, Patients, Nurses and doctors for early identification of chronic kidney disorder based on the previous cases. In order to achieve this objective knowledge was acquired from domain experts like nurses, senior medical doctors from hospitals (Jimma university referral hospital, Hawassa university referral hospital, Butajira Hospital and St. Paulose millennium hospital).

Both understood and explicit knowledge for the study was acquired from domain experts and case features were formulated. Appropriate attributes in the form of case structure which will have straight influence for decision was representing the necessary knowledge required for identifying chronic kidney disorder of patients. The system was developed by using JCOLIBRI case based framework tool which is the most compatible and reliable tool to develop case based system.

The main motivation for applying case based system over rule based system; rule based system is not efficient in making of knowledge base when there are different explanations at different time. Beside of this, the main problem of rule base system is changing and updating of knowledge base from time to time. Rule base system will not update itself to reuse the new criteria since it is only based on specific rules and facts which are restricted.

The main standards applied the symptom of kidney disorder of patients varies from time to time and person to person. Case base system use past experiences as the domain knowledge and can oftenprovide a reasonable solution through appropriate adaption. Since the system stores the new case within the existing cases, new case can be used as a case base for the next time.

This indicates that rule-based system is not operational in medical domain including chronic kidney disorder management which is extremely incomplete and context dependent to support physician in solving problems. In contrast, case-based systems use past experience as the also acknowledged. Case base system was engaged in

domain knowledge and can often provide a reasonable solution, through appropriate adaption, to these types of problems. As cases are retrieved, they may not be identical to the current case. However, when they are within some defined measure of similarity to the present case, any incompleteness and imprecision can be dealt with by a casebased reasoned. In addition, CBR does seem to address many of the shortcomings of RBS, particularly the ability of the systems to learn incrementally and to provide highly contextualized explanations.

The main aim of this research work was to apply CBR for managing chronic kidney disorder which aids physicians (Doctors, Nurses and Medical Students) in giving patients using past experiences. As we discussed the above sections, the objective was achieved. However, and as expected, improvements and open issues are still pending since there is always a room for improvement. Thus, the next tasks are to recommend some potential problem areas which are uncovered by this research.

Thus, the following recommendations have been made based on this study:

- Readiness and determination of qualities for CBR framework was done physically by thinking about which characteristic could increasingly certify and perceived in overseeing unfavorable medication response with the assistance of space specialists. The analyst proposes as future work to apply AI approach, for example, data addition attributes positioning to produce and build a lot of significant traits.

- The framework should be empowered to consequently adjust arrangements from case-base. Subsequently, the analyst recommends the investigation of different strategies of adjustment.

- The execution of the framework can be improved if a mixture approach is utilized by consolidating standards, cases and models.

- The researchers also recommended to integrate both rule based and case based reasoning for chronic kidney disorder diagnosis for purpose of achieving high performance

\section{REFERENCES}

1. WHO: 'Addressing non Communicable Diseases and Mental Health Major Challenge to Sustainable Development in the 21st Century. Discussion paper', in Editor (Ed.) $)^{\wedge}(E d s$.$) : 'Book Addressing non$ Communicable Diseases and Mental Health: Major Challenge to Sustainable Development in the 21st Century. Discussion paper' (2009 edn.), pp.

2. Dr.Girma D, Ato Dereje S, a., and S, A.W.: 'Emerging Public Health Problems in Ethiopia: Chronic Non-Communicable Diseases ', Ethiopian Public Health Association, 2012

3. Zeleke, M.: 'The Magnitude of Chronic Renal Failure and Its Associated factors among patients at St. Paulo"es Hospital, Addis Ababa, Ethiopia ', Addis Ababa University 2016

4. W, G.: 'APPLICATION OF CASE-BASED REASONING FOR ANXIETY DISORDER DIAGNOSIS ', Addis Ababa University, 2012

5. https://tenayistilign.com/2013/12/18/the-challenges-of-kidneydisease-in-ethiopia-a-call-for-diaspora-involvement/

6. Ahmed M, Begum S, F. P, N. Xiong, a., and S. A: 'Case-Based Reasoning for Diagnosis of Stress Using Enhanced Cosine and Fuzzy Similarity ', Transactions on Reasoning for Multimedia Data 2008, 1 
7. Much Aziz Muslim, Iin Kurniawati, and Sugiharti, E.: 'Expert System Diagnosis Chronic Kidney Disease Based On Mamdani Fuzzy Inference System', Journal of Theoretical and Applied Information Technology, 2015, 78, (1)

8. Akande Ruth, Amosa Babalola, Sobowale Adedayo, and M.A, H.: 'Web Based Expert System For Diagnosis And Management of Kidney Diseases', International Journal of Current Research and Academic Review, 2015 3, (2)

9. Salem, A.-B.M.: 'Case Based Reasoning Technology for Medical Diagnosis', in Editor (Ed.) ${ }^{\wedge}(E d s$.$) : 'Book Case Based Reasoning$ Technology for Medical Diagnosis' (207, edn.), pp.

10. MIKIYAS G: 'CONTENT-BASED CLASSIFICATION OF ETHIOPIAN NATIONS MUSIC VIDEO CLIP ', BAHIR DAR UNIVERSITY, 2016

11. Priti Srinivas Sajja , and Akerkar, R.: 'Knowledge-Based Systems for Development ', Advanced Knowledge Based Systems: Model, Applications \& Research 2010, 1

12. Aebissa, B.: 'Developing Knowledge based system for coffee disease diagnosis and treatment ', Addis Ababa University 2012

13. Avram, G.: 'Empirical Study on Knowledge Based Systems ', The Electronic Journal of Information Systems Evaluation 2005, 8

14. Fredlund, M.D Sillers, W.S Fredlund, a., and W. G.W.: 'Design of a knowledge-based system for unsaturated soil properties '. Proc. Third Canadian Conference on Computing in Civil and Building Engineering 1996 pp. Pages

15. S.-T. M: 'A Conceptual Model of Knowledge Elicitation '. Proc. Proceedings of the Conference on Information Systems Applied Research2009 pp. Pages

16. https://engineering.purdue.edu/ engelb/abe565/knowacq.htm

17. B, H.: 'A Case-Based Reasoning Knowledge Based System for Hypertension Management ', Addis Ababa 2011

\section{AUTHORS PROFILE}

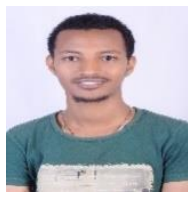

Ermiyas Birihanu Belachaw received his BSc and MSc degrees in Software Engineering and Computer Sciense, from the Adama Sciense and Technology University and Bahir Dar University , Ethiopia in 2013 and 2017, respectively. His nterests include Machine Learning, Image Processing, Software Refactoring , Design Patterns and Artifical Inteligency.

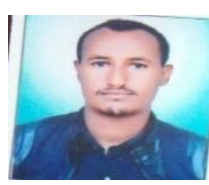

Hailemichael Kefie Tamiru received his BSc and MSc degrees in Software Engineering, from the Adama Sciense and Technology University, Ethiopia in 2013 and 2017, respectively. His interests include Software Testing and Quality Assurance ,Software Reverese Engineering, Artifical Inteligency. 